\title{
Theory and practice of feed silage in containers made of airtight film
}

\author{
Vladimir Nekrashevich ${ }^{1}$, Roman Mamonov ${ }^{2, *}$, Tatiana Torzhenova ${ }^{3}$, Irina Vorobyova $^{4}$, and Marat Khazimov $^{5}$ \\ ${ }^{1}$ Ryazan State Agrotechnological University, 390044 Ryazan, Russia \\ ${ }^{2}$ The Academy of Law Management of the Federal Penitentiary Service of Russia, 390000 Ryazan, Russia \\ ${ }^{3}$ Ryazan State Radio Engineering University named after V. F. Utkin, 390005 Ryazan, Russia \\ ${ }^{4}$ Ryazan State Medical University, 390026 Ryazan, Russia \\ ${ }^{5}$ Kazakh National Agrarian University, 050010 Almaty, Republic of Kazakhstan
}

\begin{abstract}
The article provides a brief analysis of research results in the field of preparation and storage of silage feed. A brief description of the innovative energy - resource saving technology for the preparation and storage of silage in vacuum-processed containers made from an airtight film is given. The theory of compaction of feed in a container due to its self-weight of the silage and vacuum pressure is presented. It is shown that only vacuum gauge pressure acts on the top of a container, and the difference between the gauge pressure and the lateral and axial pressure, respectively act on the side and bottom of it. The practice of using vacuum-processed containers during silage has shown that a compacted layer is formed over the entire surface of the obtained monolith, which prevents the exit of juice and air from silage. With the porosity of the mass approaching zero, the density of grinded mass of plants approaches the density of their stems and further compaction of silage is senseless. Sufficient vacuum for silage is $60-70 \mathrm{kPa}$. The density of the compressed silage monoliths in this case is $700-800 \mathrm{~kg} / \mathrm{m}^{3}$.
\end{abstract}

\section{Introduction}

One of the most important feeds in diets of farm animals is silage. It is enough to say that for cattle, for example, it comprises up to 40 , and sometimes more percent of nutritional value $[1,2]$. Nowadays the main crop for the preparation of silage is corn [3].

Analyzing modern methods of feed silage, it is necessary to note a number of significant drawbacks in their application. These include: high costs for the construction of tranches and towers; large losses of silage and nutrients, reaching 10-25\% [4], and sometimes more; high acidification of silage mass [5-7]. Most studies are devoted to the biology of silage process [5-9]. Only in recent decades, the study of the effect of vacuum on the process of silage of feed began $[10,11]$.

All the existing technologies are associated with mechanical compaction of silage mass [3]. The authors propose a technology with compaction of silage by vacuum in containers of airtight film [12].

The essence of this technology is as follows. The harvesting of green mass is carried out in the usual way: mowing, grinding and loading of grinded mass into vehicles are carried out by a forage harvester. In case study, the grinded mass is loaded into a special container made of an airtight film, which is placed in a transport bag, which, in turn, is placed and suspended in a cassette.

The container filled with a silage mass is first hermetically closed, and then air is pumped out of it by a vacuum pump, that is, it is vacuum-processed. The containers are delivered for storage using vehicles with manipulators. The feed is stored almost close to the airless environment.

\section{Materials and methods}

For the experiments, corn was chosen as the most used in agricultural production for silage of feed. Due to the fact that it is proposed to carry out the silage in sealed containers made from an airtight film, it is necessary to take into account the changes in the density and porosity of silage mass depending on the initial bulk mass of grinded feed, as well as its compaction under the influence of its self- weight and vacuum pressure.

The density of the corn stalks was determined by the pycnometric method, that is, a cut stalk was first weighed and then lowered into a measuring container with liquid, and the volume of the stalk was determined by the difference in volumes before and after immersion. The ratio of the mass of the stalk to the volume gives its density. The ratio of the mass of grinded particles of corn to the volume of the tank into which they are put gives their bulk mass. The porosity $\pi$ of the silage mass was determined by the formula:

$$
\pi=\frac{\rho-\gamma}{\rho}
$$

where $\rho$-density of corn stalks, $\mathrm{kg} / \mathrm{m}^{3}$;

* Corresponding author: mamonov.agrotexnol@yandex.ru 
$\gamma$ - bulk mass of corn particles, $\mathrm{kg} / \mathrm{m}^{3}$.

During the course of the research the authors consider the process of compaction of silage in a soft container under the action of its self- weight and vacuum. In order to simplify the theoretical study of the process, the authors apply the principle of independence of the action of forces, thus they consider separately the compaction of the material from its self- weight (Fig. 1) and vacuum and then the results are added [13].

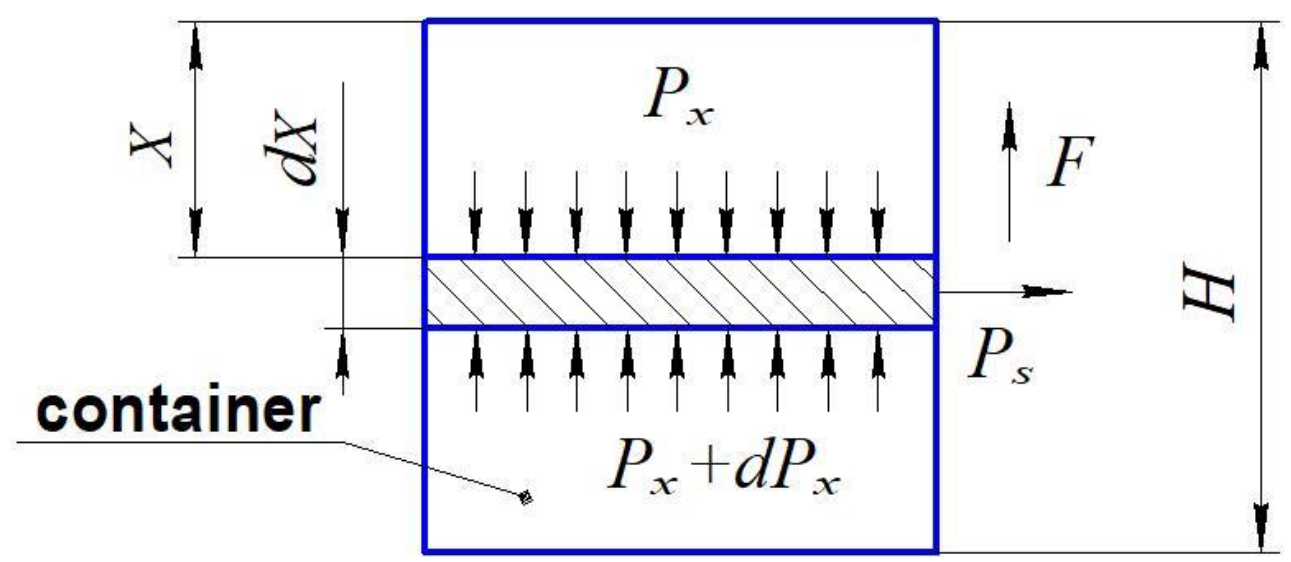

Fig. 1. Scheme of effective pressures causing compaction of the material from its self- weight.

At a distance $X$ from the top of the container, pressure will act on the elementary layer of material of thickness $d X$ :

$P_{x}$ - axial pressure from self- weight from the top;

$P_{x}+d P_{x}-$ axial pressure from self- weight from the bottom;

$P_{s}$ - lateral pressure of a thrust of material from selfweight;

$F$ - frictional force from lateral pressure.

The elementary friction force $\mathrm{F}$ is determined by the formula

$$
F=f P_{\mathrm{s}} \Pi d X
$$

where $f$-coefficient of friction of the material on the walls of the container;

$\Pi$-perimeter of the container cross section.

Lateral pressure arising under the action of its selfweight is determined using the equation

$$
P_{s}=P_{x} \xi
$$

where $\xi$ - side thrust coefficient.

The equation of equilibrium of the material layer in projection onto the vertical axis of the container is the following

$$
P_{x} S-\left(P_{x}+d P_{x}\right) S-f \xi P_{x} \Pi d X=0
$$

When the necessary transformations are made and the variables are divided, the following equation is obtained:

$$
\frac{d P_{x}}{P_{x}}=-f \xi \Pi d X / S
$$

where $S$ - container cross-sectional area

Integrating the left side of the equation in the range from $\mathrm{P}=0$ to $\mathrm{P}$, and the right from 0 to $X$ the authors define the law of distribution of axial pressure along the height of the container

$$
P_{x}=P e^{-f \xi \Pi x / s},
$$

where $P$ - axial pressure from self-weight from the bottom, or

$$
P_{x}=q g x e^{-f \xi \Pi x / S},
$$

where $q$-Density of material;

$$
g \text { - gravity factor. }
$$

The axial pressure to the mass from the top will be zero, and from the bottom it will be determined by the formula

$$
P_{c H}=q g H e^{-f \xi \Pi H / S},
$$

where $H$ - the height of the container.

For a cube, the height and length of the rib are equal to $H$; therefore, formula (7) takes the form

$$
P_{c H}=q g H e^{-f \xi 4} \text {. }
$$

For monoliths of cylindrical shape and height $\mathrm{H}$, the formula will have the form

$$
P_{c H}=q g H e^{-f \xi \Pi 4 H / d},
$$

where $\mathrm{d}$ - diameter of pressed monolith.

The authors consider the compaction of the material caused by the exposure to the vacuum. The magnitude of the vacuum pressure $\mathrm{P}_{\mathrm{v}}$ on the material is determined by the formula

$$
P_{v}=P_{a}-P_{r}
$$

where $P_{a}-$ atmospheric pressure;

$P_{r}-$ residual air pressure in the container.

Since the vacuum pressure acts on all faces of the cube volume and with the same force, and the forces of 
opposite faces are directed towards each other and of the same magnitude, therefore, in the center of the cube, the pressure caused by the vacuum will be zero.

The pressure on the upper face of the cube during vacuum processing will be equal to $P_{v}$, and the lower face will be determined by the formula

$$
R_{r S H}=P_{v}-q g a e^{-4 f \xi} .
$$

The pressure on the side faces of $R_{r s}$ is determined by the formula

$$
R_{r s}=P_{v}-q g a \xi e^{-4 f \xi}
$$

Thus, it was found that the vacuum pressure acts only on the upper face, the lower face is affected by the

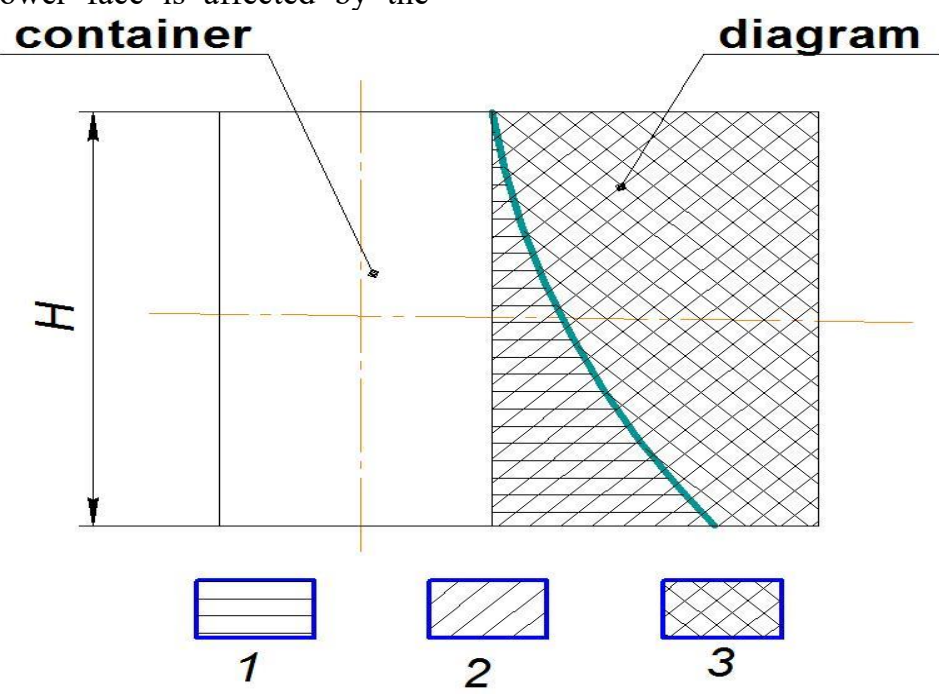

Fig. 2. Diagram of the distribution of pressures in a container with silage to the lateral face during vacuum processing: 1 - diagram of lateral pressure, 2 - diagram of vacuum pressure, 3 - resultant diagram.

\section{Results}

The results of experiments on the determination of the density of corn stalks with one or two ears, or without them, with an average length of $2.08 \mathrm{~m}$ and a mass of $0.714 \mathrm{~kg}$ showed that their density depends on humidity and ranges from 730 to $850 \mathrm{~kg} / \mathrm{m}^{3}$ with a corresponding pressure difference between the vacuum and axial pressure from gravity and the difference between the vacuum pressure and the pressure of the lateral thrust acts on the lateral faces and depends on the parameters of the container, the friction coefficients and the lateral thrust of the silage material and its density.

Figure 2 shows an approximate diagram of the distribution of pressures on the lateral face of self-weight of the material and vacuum pressure. It can be seen from the figure that the total pressure in the lower part of the container decreases by the pressure of the lateral thrust. If $P_{v}<P$, then juice may flow out of the compressed monolith, and when $P_{v}>P$, juice does not flow out, which is usually in practice. 
The graphical dependence shows that with the increase in the bulk mass of the silage culture, leads to the decrease of its porosity. The dashed line on the left indicates that, theoretically, with the bulk mass approaching zero, its porosity approaches 1 . The dashed line on the right indicates that when the bulk mass of the feed approaches the density of the stalk itself, the porosity approaches zero.

Such a phenomenon is possible only when compaction of the ground mass occurs. This indicates that all the air is displaced from the grinded mass and there is no sense in the compaction of the silage mass, as further compaction will deform the particles themselves, squeeze the juice out of them and deteriorate the quality of the feed.

The experiments under laboratory and field conditions show that when a vacuum is applied to the silage, a solid layer appears on its surface, which prevents the exit of juice and air from the formed monolith. This happens if the axial pressure is less than vacuum one.

Therefore, in order to keep the juice in a compacted monolith, it is necessary to increase the vacuum pressure as the height of the container increases. The appearance of juice at the bottom of the container may also indicate that, the deformation of particles of the silage mass occurs and juice is squeezed out of them when a vacuum is too high. Therefore, the density of silage feed can not be increased more than the density of the stalks themselves. It should be depending on the silage culture in the range from 680 to $820 \mathrm{~kg} / \mathrm{m}^{3}$.

The results of the experiments on the determination of the quality of silage from the same mass of corn harvested simultaneously showed that silage in vacuumprocessed containers contained $21.9 \%$ more protein, $20 \%$ more carotene at ph 4.3 compared to silage, prepared in tranches. The moisture content of the silage in vacuum-processed containers was slightly higher than that prepared in the tranches, which confirmed the assumptions about the pinching of a part of the juice in the silage mass during vacuum processing.

\section{Discussion}

The experiment shows that for the successful implementation of the preparation and storage of silage in vacuum-processed containers, the complex of equipment must contain: feed harvesting units, equipment for receiving green grinded mass, equipment for silage and vacuum processing of containers, loading and delivering containers in transport bags to storage places.

The results of experiments on the determination of the porosity of the silage mass showed that when the porosity of the silage mass approaches the density of the stalks from which it is made, the deformation of the particles begins, which leads to the release of juice from them. Therefore, when vacuum processing the silage mass there is no sense to exceed the vacuum value more than the permissible one in order to compact it.
It was theoretically established that compaction of the silage mass under the action of its self-weight follows exponential law and depends on the height of the fill in the container, the friction coefficients and the lateral thrust.

The compaction under the action of vacuum acts volumetrically and therefore only vacuum pressure acts in the container on top of the compacted monolith and the difference between the vacuum pressure and, respectively, axial and lateral acts only from the bottom and sides. Empirically it was established that during vacuum processing, a compacted layer is formed on the surface of the monolith, which prevents the exit of juice and air from its middle. At a vacuum gauge below axial pressure, juice can exit from the middle of the monolith, which is also empirically confirmed.

\section{Conclusion}

Thus, the above mentioned complex of equipment allows the preparation of grinded green mass, loading it into containers of airtight film, silage and vacuum processing of containers, and delivering containers with silage mass to storage places. It was theoretically revealed that the compaction of the silage mass can be carried out both by means of its self-weight and vacuum. It was established that it is not advisable to conduct compaction of the grinded silage mass above the density of the stalks themselves due to increased energy costs and increased juice production. A sufficient vacuum pressure for corn silage is $60-70 \mathrm{kPa}$. In this case, the silage is of high quality.

\section{References}

1. A.P. Kalashnikov, Norms and diets for feeding farm animals: a reference guide (Nauka, Moscow, 2003)

2. S.N. Khokhrin, Feeds and feeding animals (Lan, St. Petersburg, 2002)

3. L.G. Boyarsky, Feed technology and full-fledged feeding of farm animals (Phoenix, Rostov-on-Don, 2001)

4. T. Keller, H. Nonn, H. Jeroch, The effect of sealing and of additives on the fermentation characteristics and mould and yeast counts in stretch film wrapped big-bale Lucerne silage, Arch. Tierernahr., 51 (1998)

5. J.M. Asplund, Principles of Protein Nutrition of Ruminants (CRC press, Boca Raton, FL, 1994)

6. R. Erdman, Silage Fermentation Characteristics Affecting Feed Intake, in Proc. National Silage Product. Conf., vol. 210 (Syracuse, New York, 1993)

7. J.H. Harrison, R. Blauwiekel, M.R. Stokes, Fermentation and utilization of grass silage, J. Dairy Sci., 77 (1994)

8. L. Kung, R. Shaver, Interpretation and use of silage fermentation analysis reports, Focus on Forage, 3, 13 (University of Winconsing Extrnsion, 2001) 
9. P. McDonald, N. Henderson, S. Heron, The Biochemistry of Silage (Chalcombe Publications, UK, 1991)

10. D.V. Ivanov, Modes and technical means of preparing silage crops in packages with reduced pressure of gas medium (Theses, Stavropol, 2010)

11. H.E. Johnson, R.J. Merry, D.R. Davies, D.B. Kell, M.K. Theodorou, G.W. Griffith, Vacuum packing: $a$ model system for laboratory-scale silage fermentations, J. of Appl. Microbiol. (2005)
12. V.F. Nekrashevich, I.V. Vorobyova, K.S. Afanasyeva, M.A. Borontova, A.E. Belov, Preparation and storage of silage in soft vacuum processed containers from an airtight film, Feed production, 10 (2017)

13. V.F. Nekrashevich, A.S. Popov, K.S. Afanasyeva, The use of vacuum during the compaction of silage in containers from airtight film, Bull. of the Ulyanovsk Agricult. Acad., 3 (2017) 